7 Abstract parent asteroids. Ammoniated ice, First order reversal curve diagrams

\title{
Constraints on the ice composition of carbonaceous chondrites from their magnetic mineralogy
}

\author{
Sanjana Sridhar ${ }^{\mathrm{a}, *}$, James F. J. Bryson ${ }^{\mathrm{a}}$, Ashley J. King ${ }^{\mathrm{b}}$, Richard J. Harrison ${ }^{\mathrm{c}}$ \\ ${ }^{a}$ Department of Earth Sciences, University of Oxford, Oxford, OX1 3AN, UK \\ ${ }^{b}$ Department of Earth Sciences, Natural History Museum, London, SW7 5BD, UK \\ ${ }^{c}$ Department of Earth Sciences, University of Cambridge, Cambridge, CB2 3EQ, UK
}

Carbonaceous chondrites experienced varying degrees of aqueous alteration on their parent asteroids, which influenced their mineralogies, textures, and bulk chemical and isotopic compositions. Although this alteration was a crucial event in the history of these meteorites, their various alteration pathways are not well understood. One phase that formed during this alteration was magnetite, and its morphology and abundance vary between and within chondrite groups, providing a means of investigating chondrite aqueous alteration. We measured bulk magnetic properties and first-order reversal curve (FORC) diagrams of CM, CI, CO, and ungrouped C2 chondrites to identify the morphology and size range of magnetite present in these meteorites. We identify two predominant pathways of aqueous alteration among these meteorites that can be distinguished by the resultant morphology of magnetite. In WIS 91600, Tagish Lake, and CI chondrites, magnetite forms predominantly from Fe-sulfides as framboids and stacked plaquettes. In $\mathrm{CM}$ and $\mathrm{CO}$ chondrites, $<0.1 \mu \mathrm{m}$ single-domain (SD) magnetite and 0.1-5 $\mathrm{m}$ vortex (V) state magnetite formed predominantly via the direct replacement of metal and Fe-sulfides. After ruling out differences in temperature, water:rock ratios, terrestrial weathering effects, and starting mineralogy, we hypothesise that the primary factor controlling the pathway of aqueous alteration was the composition of the ice accreted into each chondrite group's parent body. Nebula condensation sequences predict that the most feasible method of appreciably evolving ice concentrations was the condensation of ammonia, which will have formed a more alkaline hydrous fluid upon melting, leading to fundamentally different conditions that may have caused the formation of different magnetite morphologies. As such, we suggest that WIS 91600, Tagish Lake, and the CI chondrites accreted past the ammonia ice line, supporting a more distal or younger accretion of their

Keywords: Carbonaceous chondrites, Aqueous alteration, Magnetite framboids, Fluid composition, 


\section{Introduction}

\footnotetext{
* Corresponding author

Email address: sanjana.sridhar@univ.ox.ac.uk (Sanjana Sridhar)

Twitterhandle:@nikki_sridhar
}

The carbonaceous chondrites (CCs) are a class of undifferentiated meteorites that underwent varying extents of aqueous alteration on their parent asteroids (Brearley, 2006; Rubin et al., 2007; Howard et al., 2015; King et al., 2015). As such, these meteorites represent a unique archive of the effects of one of the key processes that influenced the textures, mineralogies, and isotopic and elemental compositions of asteroids over their lifetimes. The carbonaceous chondrites are of particular interest due to their high volatile element abundances and isotopic similarity to Earth's surface volatile elements, which has led to them being proposed to have played a significant role in the delivery of volatile elements to Earth and the other terrestrial planets (Alexander et al., 2018b). Despite the central role that aqueous alteration played on the properties of these meteorites, the mechanisms and conditions of chondrite aqueous alteration are not completely understood.

The CCs are thought to have accreted beyond the orbit of proto-Jupiter ( 3 AU) (Desch et al., 2018), and are aggregates of sub-millimeter-sized solids, including: chondrules, metallic Fe-Ni grains, and refractory inclusions (including calcium-aluminium inclusions and amoeboid olivine aggregates) all set in a fine-grained matrix. The accretion of ice during the formation of chondrite parent bodies, and its subsequent melting due to heat released by the radioactive decay of ${ }^{26} \mathrm{Al}$ and impacts (King et al., 2021b) provided the hydrous fluid responsible for the aqueous alteration recorded by these meteorites (Brearley, 2006). Variations in the extent of aqueous alteration are observed both within and between the different carbonaceous chondrite groups. The CM and CR chondrites range from mildly to extensively altered (Rubin et al., 2007; Harju et al., 2014; King et al., 2017), while the CI chondrites are all essentially fully altered (King et al., 2015). Conversely, the $\mathrm{CO}$ chondrites experienced limited aqueous alteration with many considered to be pristine (Davidson et al., 2019a).

During aqueous alteration, hydrous fluid reacted with the primitive chondrite matrix, leading to the formation of a secondary mineral assemblage. Minerals formed during this process include phyllosilicates, carbonates, Fe-sulfides, and magnetite (Brearley, 2006; King et al., 2017). These minerals can be used to investigate the process of aqueous alteration because their formation is directly affected by the pathway and conditions of these reactions.

Magnetite formed during aqueous alteration through the progressive oxidation of Fe-metal and Fesulfides. King et al. (2017) showed that within CM chondrites, the amount of magnetite formed increases with the extent of aqueous alteration. Conversely, studies of CO chondrites, which potentially represent 


\begin{tabular}{cc}
\hline Domain state & Size range $(\boldsymbol{\mu m})$ \\
\hline \hline Multi Domain & $\sim>5$ \\
Vortex & $\sim<0.1-5$ \\
Single Domain & $<0.1$ \\
\hline \hline
\end{tabular}

Table 1: Summary of the size ranges of different magnetic domain states in eqidimensional magnetite grains (Roberts et al., 2018).

primitive unaltered CM-like material (Schrader and Davidson, 2017), demonstrate that the most pristine $\mathrm{CO}$ chondrites exhibit magnetite modal abundances that far exceed those of CM chondrites, despite the minimal aqueous alteration experienced by CO chondrites (Alexander et al., 2018a). Interestingly, CO chondrites that experienced slightly more thermal metamorphism (petrological grade $\geq 3.2$ ) contain less magnetite, indicating that the preservation and abundance of magnetite is sensitive to both the amount of aqueous alteration and the conditions of metamorphism (Rubin and Li, 2019). Previous studies have shown that magnetite exists in a range of morphologies in different carbonaceous chondrite groups, including as enigmatic framboids and plaquettes in CR chondrites (Harju et al., 2014), CI chondrites (Kerridge et al., 1979; Brearley, 2006), the ungrouped C2 chondrite (C2-ung) Tagish Lake (Greshake et al., 2005), and the anomalous CM chondrite (CM-an) WIS 91600 (Brearley, 2004).

Because the formation of magnetite in CCs is controlled by the process of aqueous alteration, changes in magnetite abundance and morphology are expected to reflect variations in the conditions and pathways of aqueous alteration on their parent bodies. These differences in magnetite abundance and morphology can impact the bulk magnetic properties of these meteorites, such that magnetic measurements can provide a novel means of exploring their aqueous histories.

A unique and informative set of bulk magnetic measurements that are particularly suited for this purpose are first-order reversal curve (FORC) diagrams. The shape and intensity of a FORC diagram reflects the size, morphology, and spatial distribution of the magnetic grains in a sample, and can be considered as a fingerprint for a given type of magnetic characteristic (Roberts et al., 2014). The magnetic domain state of a grain is determined by the alignment of the magnetic moments within it, and is sensitive to grain size and morphology (Table 1) (Roberts et al., 2018; Harrison et al., 2019). As such, FORC diagrams are particularly useful for identifying the domain state of magnetic grains present. FORC diagrams of samples that contain mixtures of magnetic particles with different characteristics can be unmixed into their end-member (EM) components using principle component analysis (PCA) (Harrison et al., 2018). FORC diagrams are also able to identify the presence of sub-micron-scale magnetite that is challenging to image using scanning electron microscopy (SEM). Finally, FORC diagrams provide a means of examining the magnetic mineralogy of 


\begin{tabular}{|c|c|c|c|}
\hline Name & Type & Classification & Weathering grade \\
\hline Paris * & CM & 1.7 & W0 \\
\hline LEW 85311 & $\mathrm{CM}$ & 1.7 & $\mathrm{Be}$ \\
\hline DOM $03182 *$ & $\mathrm{CM}$ & 1.7 & B \\
\hline GRA $06172 *$ & $\mathrm{CM}$ & 1.7 & B \\
\hline LAP 04565 & $\mathrm{CM}$ & 1.6 & B \\
\hline LAP 04796 & $\mathrm{CM}$ & 1.6 & $\mathrm{~A} / \mathrm{B}$ \\
\hline LAP 04514 & $\mathrm{CM}$ & 1.6 & B \\
\hline Murchison (1) & $\mathrm{CM}$ & 1.5 & Fall \\
\hline Murchison (2) & $\mathrm{CM}$ & 1.5 & Fall \\
\hline Murchison (3) & $\mathrm{CM}$ & 1.5 & Fall \\
\hline Jbilet Winselwan & $\mathrm{CM}$ & 1.5 & W1 \\
\hline LAP 031214 & $\mathrm{CM}$ & 1.5 & B \\
\hline MCY 05231 & $\mathrm{CM}$ & 1.5 & B \\
\hline Santa Cruz & $\mathrm{CM}$ & 1.4 & Fall \\
\hline LAP 02333 & $\mathrm{CM}$ & 1.4 & B \\
\hline MIL 090288 & $\mathrm{CM}$ & 1.2 & $\mathrm{Be}$ \\
\hline LAP 031166 & $\mathrm{CM}$ & 1.2 & B \\
\hline NWA 8534 & $\mathrm{CM}$ & 1.2 & Low \\
\hline GRO 95645 & $\mathrm{CM}$ & 1.2 & $\mathrm{~B} / \mathrm{Ce}$ \\
\hline NWA 4765 & $\mathrm{CM}$ & 1.2 & Low \\
\hline LAP 02277 & $\mathrm{CM}$ & 1.2 & A \\
\hline Moapa Valley & $\mathrm{CM}$ & 1.1 & Low \\
\hline MIL $05137^{\mathrm{a}}$ & $\mathrm{CM}$ & 1.1 & $\mathrm{Be}$ \\
\hline MIL $07689^{\text {a }}$ & $\mathrm{CM}$ & 1.1 & $\mathrm{C}$ \\
\hline Ivuna (1) & CI & 1.0 & Fall \\
\hline Ivuna (2) & CI & 1.0 & Fall \\
\hline Orgueil (1) a & CI & 1.0 & Fall \\
\hline Orgueil (2) & CI & 1.0 & Fall \\
\hline WIS 91600 (1) & CM-an & $\sim 1.4$ & $\mathrm{~A} / \mathrm{Be}$ \\
\hline WIS $91600(2)$ & CM-an & $\sim 1.4$ & $\mathrm{~A} / \mathrm{Be}$ \\
\hline DOM 08006 & $\mathrm{CO}$ & 3.0 & $\mathrm{~B} / \mathrm{C}$ \\
\hline MIL 090010 & $\mathrm{CO}$ & 3.0 & $\mathrm{~A} / \mathrm{B}$ \\
\hline
\end{tabular}

Table 2: Petrographic description of the chondrites examined in this study. Classification of aqueous alteration grade from Howard et al. (2015); King et al. (2015); Alexander et al. (2018a); King et al. (2019) and Lee et al. (2019) based on phyllosilicate fraction. Weathering grade identified from the Meteoritical Bulletin; if samples were seen to fall this has been indicated. Chondrites whose phyllosilicate and magnetite abundances were established by unpublished data are indicated by an asterisk "*". Chondrites which were imaged by scanning electron microscopy are indicated by the superscript "a".

the entire volume of a sample quickly and non-destructively. Here, we use FORC diagrams to recover quantitative constraints on the proportions of the different types of magnetic grains that formed in a suite of CCs that experienced different extents of aqueous alteration. We use this to constrain the pathways of

s8 aqueous alteration experienced by various CCs. We examine the possible reasons for the different styles and

s9 pathways of aqueous alteration and the potential link between ice chemistry and the evolution of chondrite

эo parent bodies. 


\section{Methods}

\subsection{Sample petrology}

Bulk magnetic measurements were conducted on $22 \mathrm{CM}$, two CI, and two CO chondrite powders. The same powders were previously characterised using infrared (IR) spectrocopic analysis by Bates et al. (2020), and X-ray diffraction (XRD) by King et al. (2015, 2017, 2019, 2021a) who assigned each meteorite a petrologic type based on its phyllosilicate abundance (e.g. Howard et al. (2015)). Except for Paris, LEW 85311, DOM 03182, GRA 06172, Murchison (1), Santa Cruz, Ivuna (1), and Orgueil (1), all the powders were also artificially heated to $150^{\circ} \mathrm{C}$ under vacuum for IR spectroscopic measurements (Bates et al., 2020).

In addition, we studied two millimetre-sized chips of the CM-an chondrite WIS 91600. Although the official classification of WIS 91600 is CM-an, following the assessment of Rubin et al. (2007) we do not consider it to be a CM, rather an ungrouped C2 chondrite. Both WIS 91600 and Jbilet Winselwan show evidence of impact heating on their parent asteroids at peak temperatures of $<600^{\circ} \mathrm{C}$ and $400-500^{\circ} \mathrm{C}$, respectively (Bryson et al., 2020a; King et al., 2019, 2021b). The two CO chondrites studied here, DOM 08006 and MIL 090010, have undergone extremely low degrees of aqueous alteration (Davidson et al., 2019a) which led to them containing relatively high amounts of magnetite and makes them comparatively different to most members of this group (which typically contain several wt\% FeNi metal instead (Rubin and Li, 2019)). A complete list of the chondrites examined, and their petrologic type and weathering grade is given in Table 2. Though we cannot rule out larger scale sample heterogeneity, we homogenised our powders so we are confident that we created representative subsamples of the original chips of each meteorite.

\subsection{Bulk magnetic properties}

A MicroMag 2900 Series Alternating Gradient Magnetometer (AGM) was used to measure the magnetic properties of the samples. Hysteresis loop measurements were conducted using an applied saturation field of $1.5 \mathrm{~T}$. The averaging time was $100 \mathrm{~ms}$ and the field increment was $6 \mathrm{mT}$. Paramagnetic adjustment was conducted to remove the overprint of paramagnetic behaviour in the samples.

\subsection{Sample Preparation}

Hysteresis loop measurements were conducted on powdered samples loaded into $5 \mathrm{~mm}$ diameter gelatine capsules. Sample masses are included in Table S3 in the supplementary information. FORC measurements were conducted on immobilised powder samples; $<1 \mathrm{mg}$ of the powdered sample was placed on a $5 \mathrm{~mm}$ diameter glass disk which was coated with a thin film of Bostik superglue. This was covered by another 
drop of superglue to fully encase and immobilise the powder. Samples were mounted onto the AGM using a perpendicular mounting rod and were held in place by silicon grease.

\subsection{FORC diagrams}

We measured 300 FORCs per sample with a $2.09 \mathrm{mT}$ field-step size, a $275 \mathrm{~ms}$ averaging time, and a saturating field of $1 \mathrm{~T}$. The FORC diagrams were processed using the VARIFORC approach (Egli, 2013) within the FORCinel software package (Harrison and Feinberg, 2008). During smoothing, we used a value of 0.2 for the horizontal and vertical lambda values $(\lambda)$. The full range of smoothing factors used is included in the supplementary information (Table S1).

\subsection{Principle component analysis}

Principle component analysis (PCA) was conducted on the processed FORC diagrams of the CM, CI and C2-ung chondrites, using the FORCem software package (Harrison et al., 2018). This analysis recovers the proportions of the principle components that describe the variation among the measured FORC diagrams. Because the FORC diagrams of different magnetic grain types are distinct, they occupy specific points in principle component space. FORC diagrams of samples that are mixtures of magnetic grain types are themselves a mixture of FORC signals of the different magnetic grains (as seen in Fig. S1), and can be "unmixed" to recover the proportions of each end-member that contributes to the bulk FORC signal.

In our analysis, physically realistic end-members were chosen to identify the magnetic domain states that mix to generate the magnetic signal of our samples. These end-members were chosen on the criteria that: (i) their generated hysteresis loop saturated at $1 \mathrm{~T}$; (ii) the arms of the generated hysteresis loop did not intersect; and (iii) the FORCs are monotonic (Harrison et al., 2018). Feasibility metric contours are included on the PCA score plots to highlight the regions of PCA space where realistic EMs can be found. A value of 1 indicates that all the criteria have been satisfied, and a value of 0 indicates that they are completely unsatisfied.

PCA was conducted on two subsets of chondrites that each experienced similar levels of aqueous alteration: the first PCA was conducted on chondrites of petrological type 1.0-1.2; and the second PCA was conducted on chondrites of petrological type 1.3-1.7. Splitting the chondrites into these groups based on their extent of aqueous alteration allowed for trends between the groups to be easily identified due to the large diversity of FORC characteristics displayed among CM chondrites.

\subsection{Magnetite modal abundance}

The magnetite volume fraction present in each gel cap sample was calculated using: 


$$
\text { Magnetite }(\text { vol } \%)=\frac{\frac{M_{S}}{\text { samplemass }} \times \rho_{C C}}{480000 A m^{-1}} \times 100
$$

Where $\mathrm{M}_{\mathrm{S}}$ is the saturation magnetisation of the chondrite, $\rho_{C C}$ is the grain density of the powders $(\mathrm{CM}$, WIS $91600=2900 \mathrm{kgm}^{-3}, \mathrm{CI}=2400 \mathrm{kgm}^{-3}, \mathrm{CO}$ (falls) $=3400 \mathrm{kgm}^{-3}$ ) (Macke et al., 2011), and 480000 $\mathrm{Am}^{-1}$ is the $\mathrm{M}_{\mathrm{S}}$ per unit volume of magnetite. This method assumes that all the magnetisation of the sample is due to magnetite, which is unlikely to be the case as previous XRD studies show the presence of metal and sulfide (including pyrrhotite), and an unknown fraction of the sulfide may be magnetic (Howard et al., 2015; King et al., 2017; Alexander et al., 2018a). To account for this, we calculated the representative error that could be attributed to magnetisation due to metal and magnetic pyrrhotite (see supplementary information).

\subsection{Scanning electron microscopy}

Scanning electron microscopy and energy-dispersive X-ray spectroscopy (SEM-EDS) analysis was undertaken on powdered samples and carbon-coated thin sections to image magnetite morphologies in different chondrites. Powdered samples were immobilised by placing the powder onto a drop of acetone, which evaporated to leave the powder adhered to the surface of an aluminium disk. Secondary electron (SE) and back-scattered electron (BSE) images were acquired, and energy-dispersive X-ray spectroscopy (EDX) was carried out using a QEMSCAN 650F.

\section{Results}

\subsection{Magnetite abundance}

A plot of the magnetite abundance determined from magnetic measurements compared to the magnetite abundance recovered from XRD measurements shows a positive correlation (Fig. 1a). The magnetite abundance recovered from XRD are generally greater than that recovered from magnetic measurements, though care should be taken when making direct comparisons due to the potentially heterogeneous nature of chondrite powders. A plot of the calculated magnetite fraction in CM chondrites against the phyllosilicate fraction reported in the literature does not appear to show a correlation (Fig. 1b). The full list of the bulk magnetic parameters measured for each sample can be found in the supplementary information (Table S3).

\subsection{Day plot}

A plot of hysteresis parameters $\mathrm{M}_{\mathrm{rs}} / \mathrm{M}_{\mathrm{s}}$ vs $\mathrm{H}_{\mathrm{cr}} / \mathrm{H}_{\mathrm{c}}$ (a Day plot) suggests that the CO, CM, CI, and C2-ung chondrites are dominated by pseudo-single domain (PSD) grains that tend to be $<0.1-5 \mu \mathrm{m}$, and 

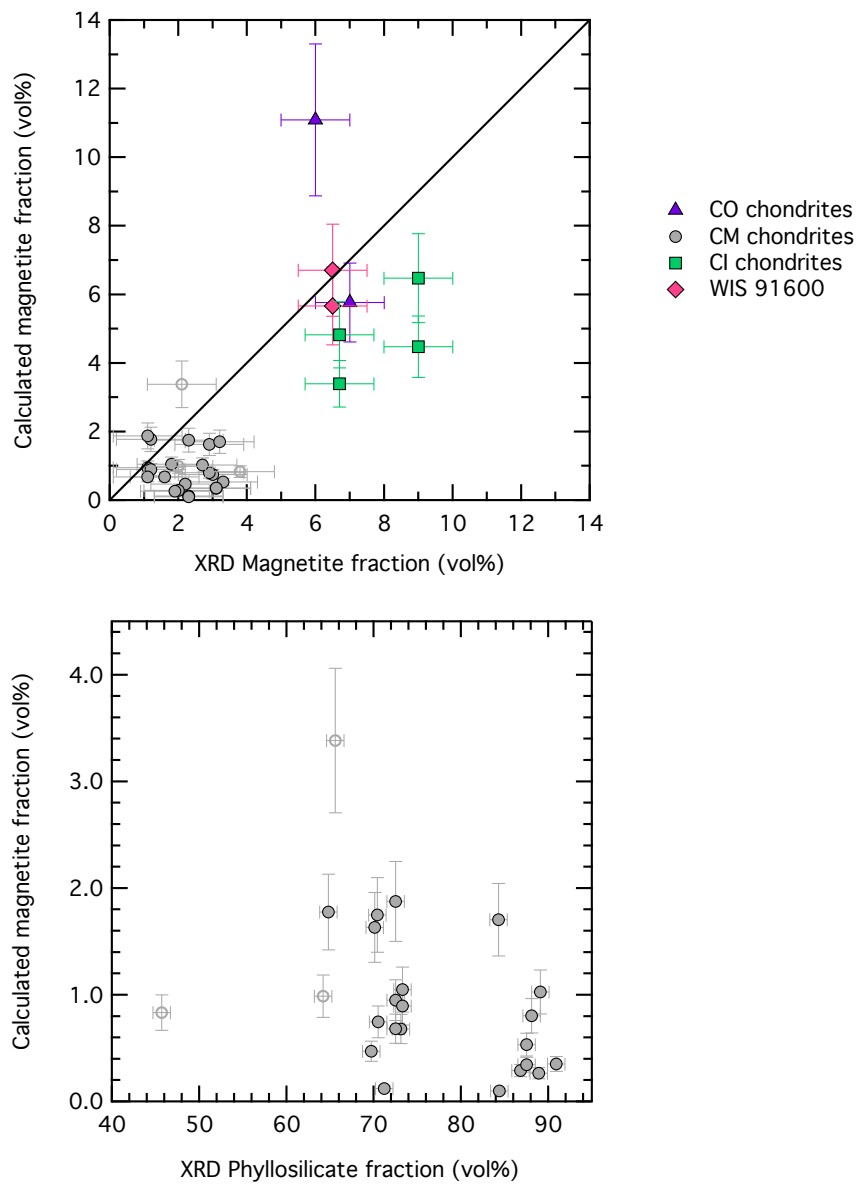

Figure 1: a) Magnetite fraction of the CM, CI, CO and C2-ung chondrites calculated via magnetic measurements plotted against magnetite fraction measured using XRD. A 1:1 line is shown for better comparison between the abundances recovered from XRD and those from this study. b) Magnetite fraction of the CM chondrites calculated via magnetic measurements plotted against phyllosilicate fraction measured using XRD. Phyllosilicate and magnetite XRD data from Alexander et al. (2018a); Howard et al. (2015); King et al. (2015, 2017, 2019, 2021a) and Lee et al. (2019). Data for the CM1.7 chondrites Paris, DOM 03182 and GRA 06172 are unpublished and are represented by unfilled points.

superparamagnetic-single domain grains (SP-SD) that tend to be $<0.1 \mu \mathrm{m}$ (Fig. 2). Plotting bulk hysteresis parameters in this way does not reveal any systematic variation with degree of aqueous alteration.

\subsection{FORC diagrams}

FORC diagrams of the CO 3.0 chondrite DOM 08006, the CM 1.7 chondrite Paris, the CM 1.5 chondrite Murchison, and CM 1.1 chondrite MIL 05137, the CI chondrite Orgueil, and the C2-ung chondrite WIS 91600 are shown in Fig. 3. The remaining FORC diagrams can be found in the supplementary information.

\subsection{1. $C O$ chondrites}

The FORC diagrams of $\mathrm{CO}$ chondrites display a tri-lobed pattern with a central peak (Fig. 3a). One lobe extends along the positive $\mathrm{B}_{\mathrm{u}}$-axis, one spreads along the $\mathrm{B}_{\mathrm{c}}$-axis, and the third spreads diagonally 


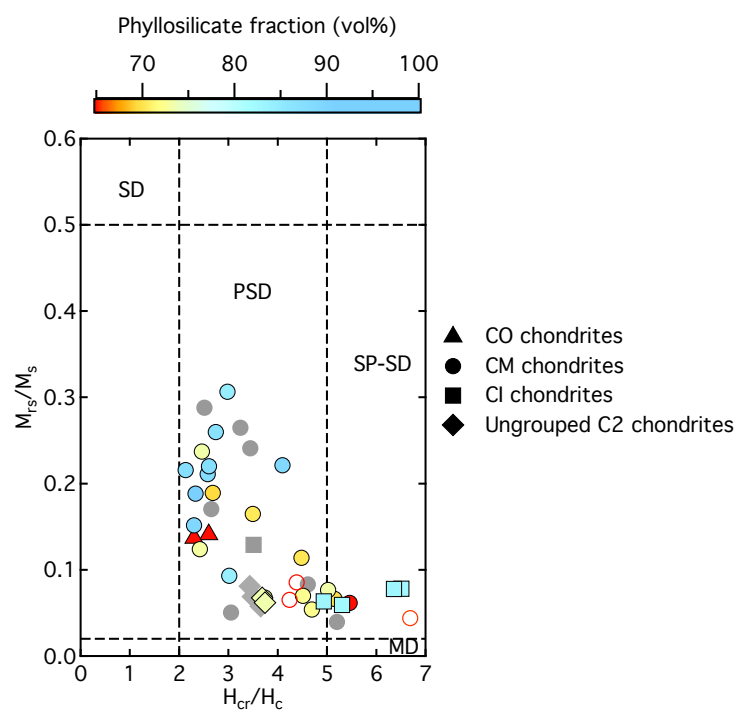

Figure 2: Day plot of all chondrite samples. Each meteorite is colour coded based on their phyllosilicate fraction measured using XRD. There is no correlation between domain state of the magnetite grain and their extent of aqueous alteration. Phyllosilicate XRD data from Alexander et al. (2018a); Howard et al. (2015); King et al. (2015, 2017, 2019, 2021a) and Lee et al. (2019). Data for the CM1.7 chondrites Paris, DOM 03182 and GRA 06172 are unpublished and are represented by unfilled points. Literature data from Cournède et al. (2015) and Thorpe et al. (2002) are also plotted in grey (excluding the CM chondrites Murray and Paris as their values of $\mathrm{H}_{\mathrm{cr}} / \mathrm{H}_{\mathrm{c}}$ are too large)

downwards in the positive $\mathrm{B}_{\mathrm{c}}$-axis and negative $\mathrm{B}_{\mathrm{u}}$-axis direction. The peak intensity is at a slightly negative $\mathrm{B}_{\mathrm{u}}$ value at a $\mathrm{B}_{\mathrm{c}}$ value of $\sim 25 \mathrm{mT}$. This signature is characteristic of isolated vortex state $(\mathrm{V})$ grains that are typically $<0.1-5 \mu \mathrm{m}$ in size when equidimensional magnetite (Roberts et al., 2014). Spreading along the $\mathrm{B}_{\mathrm{u}}$-axis is due to the self-demagnetizing field generated by the magnetic grains.

\subsection{2. $C M$ chondrites}

The FORC diagrams of $\mathrm{CM}$ chondrites display variable patterns, with some chondrites (e.g. CM 1.1 MIL 05137) displaying a more prominent tri-lobed pattern with a central peak, characteristic of isolated vortex state grains, while others (e.g. CM 1.5 Murchison) display a strong signal along the central ridge, characteristic of isolated single domain (SD) grains of magnetite, typically $<0.1 \mu \mathrm{m}$ in diameter (Harrison and Lascu, 2014). Asymmetric vertical spreading along the $\mathrm{B}_{\mathrm{u}}$-axis in FORC diagrams of SD particles indicates weak interactions and/or some vortex state contribution. Rare CM chondrites, such as the CM 1.7 Paris (Fig. 3f), display a peak intensity on the origin, and symmetric vertical spreading along the $\mathrm{B}_{\mathrm{u}}$-axis, characteristic of larger, multi-domain (MD) grains (Roberts et al., 2014). The FORC diagrams of the CM chondrites Moapa Valley, LAP 031166, NWA 4765, MIL 090288, LAP 02277, and LAP 03124 (Fig. S2 and S4) show a weak signal extending along the $\mathrm{x}$-axis past $300 \mathrm{mT}$, indicating the presence of some higher 

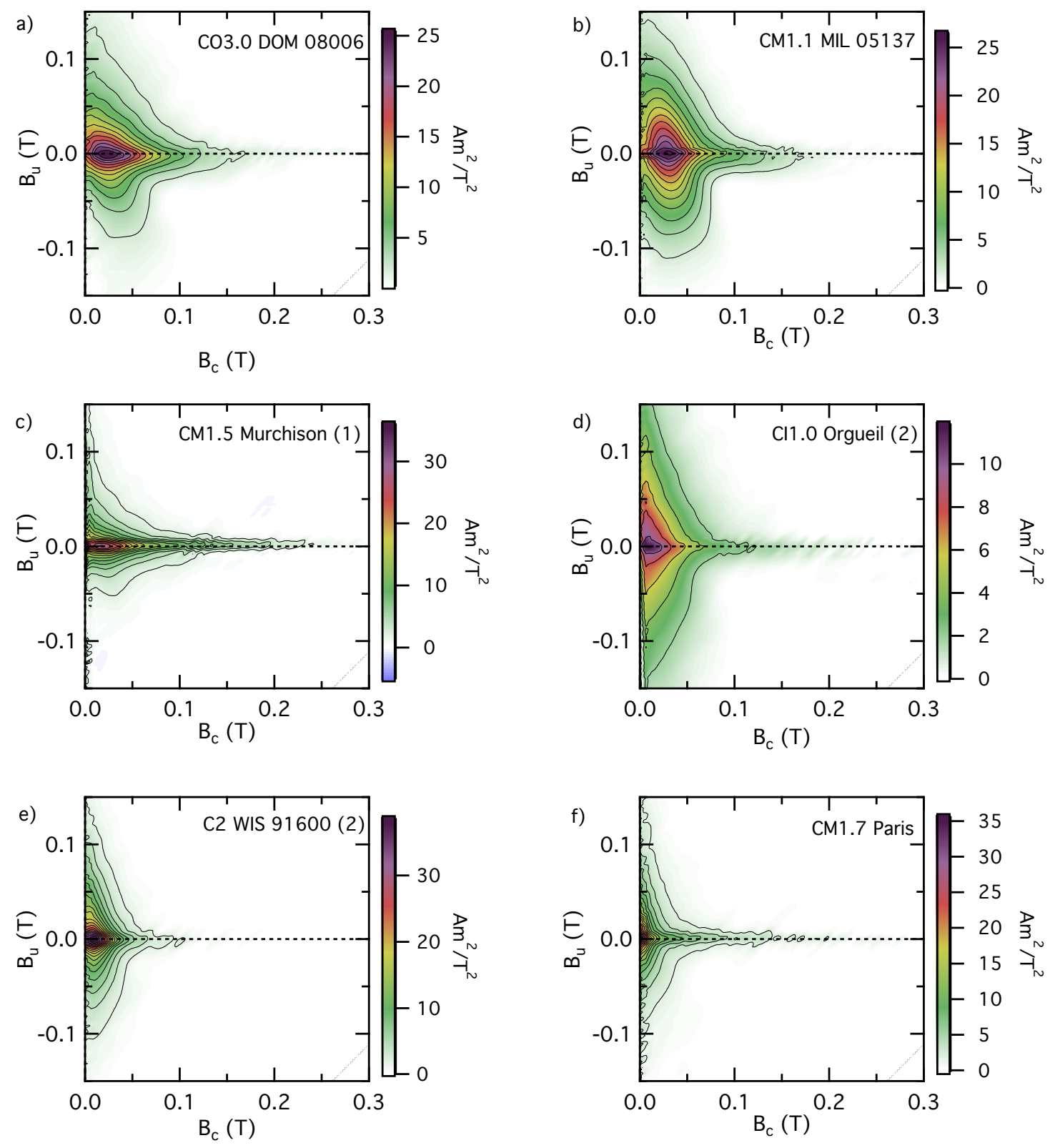

Figure 3: FORC diagrams of a) CO3.0 DOM 08006, b) CM1.1 MIL 05137, c) CM1.5 Murchison (1), d) CI1.0 Orgueil (2), e) C2 WIS 91600, and f) CM1.7 Paris. The colour scaling of intensity and the contour intervals differ between each plot to best highlight the features present.

coercivity grains (e.g., pyrrhotite).

\subsubsection{CI chondrites}

The FORC diagrams of CI chondrites are triangular in shape, with peak intensity off-centre along the $\mathrm{B}_{\mathrm{c}^{-}}$ axis, at $10 \mathrm{mT}$. The FORC signal extends vertically along the $\mathrm{B}_{\mathrm{u}}$-axis to $\pm 200 \mathrm{mT}$, and horizontally along 
the $\mathrm{B}_{\mathrm{c}}$-axis mostly to $100 \mathrm{mT}$, though there is evidence for a weak signal up to $200 \mathrm{mT}$. This signature is characteristic of closely packed, interacting vortex/multi domain grains, often found in framboids (Harrison et al., 2019). These equidimensional magnetite grains are typically $<1 \mu \mathrm{m}$ in size.

\subsubsection{C2-ung chondrites}

The FORC diagrams of WIS 91600 also display a triangular shape, with peak intensity off-centre along the $\mathrm{B}_{\mathrm{c}}$-axis, at $15 \mathrm{mT}$. The vertical spreading is constrained to $\pm 100 \mathrm{mT}$, while intensity along the $\mathrm{B}_{\mathrm{c}}$-axis extends to $50 \mathrm{mT}$. This is characteristic of clustered, interacting SD/vortex state grains, $<1 \mu \mathrm{m}$ in size.

\section{4. $P C A$}

PCA of the CM and C2-ung chondrites with petrological grades between 1.3-1.7 are shown in Fig. 4, along with the identified end members. PCA of CM and CI chondrites with petrological grades 1.0-1.2 are shown in Fig. 5, along with the identified end members. The calculated proportions of each principle component and end-member present in each chondrite are listed in the supplementary information (Table S2). These end-member proportions represent the calculated proportions of the different morphologies of magnetite present in each of the measured samples.

\subsubsection{Aqueous alteration grade 1.3-1.7}

Two principle components that accounted for $81 \%$ of the variability were identified and three endmembers were picked manually to describe the variation among samples with aqueous alteration grades between 1.3-1.7. End-member 1 (EM1) displays a strong central ridge, with a peak intensity along the $\mathrm{B}_{\mathrm{c}}$-axis at $40 \mathrm{mT}$. The signal extends along the $\mathrm{B}_{\mathrm{c}}$-axis, to a coercivity value of $\sim 250 \mathrm{mT}$, with a pronounced negative signal below and to the left of the central ridge. The slight teardrop shape indicates weak interactions between individual magnetite grains. This is characteristic of weakly interacting, single domain (SD) grains (Harrison and Lascu, 2014). End-member 2 (EM2) displays a concentrated vertical spreading along the $\mathrm{B}_{\mathrm{u}}$-axis, characteristic of MD grains (Roberts et al., 2014). EM2 also contains a pronounced central ridge extending along the $\mathrm{B}_{\mathrm{c}}$-axis to $200 \mathrm{mT}$ due to a residual SD component. End-member 3 (EM3) displays a weak triangular pattern, resembling interacting V/MD grains (Harrison et al., 2019). The signal spreads vertically along the $\mathrm{B}_{\mathrm{u}}$-axis to $\pm 100 \mathrm{mT}$, and the peak lies slightly off the origin along the $\mathrm{B}_{\mathrm{c}}$-axis.

The C2-ung chondrite WIS 91600 plots in distinct principle component space compared to the CM1.3-1.7 chondrites. WIS 91600 defines EM3, indicating the presence of interacting V/MD grains in this meteorite. The CM1.3-1.7 chondrites plot between EM1 and EM2, suggesting that they contain a mixture of SD and MD grains with varying proportions. 


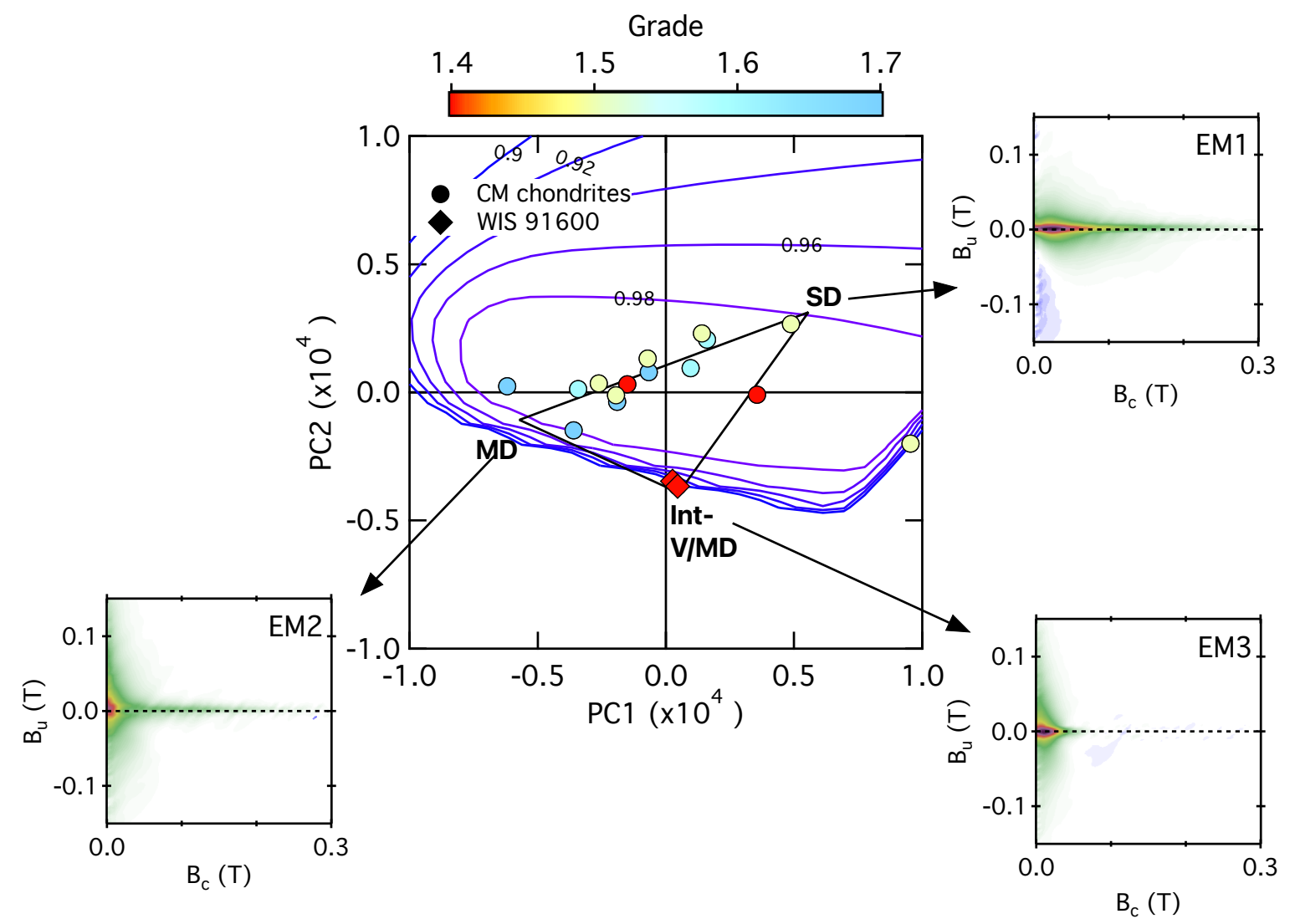

Figure 4: Score plot displaying the proportions of PC1 and PC2 present in each CM and C2-ung chondrite of aqueous alteration grade 1.3-1.7. The positions of the end members are also displayed. EM1 represents non-interacting, SD magnetite; EM2 represents interacting, MD magnetite grains; and EM3 represents interacting V/MD grains. The intensity scaling differs between each end member to better highlight their features. Contours of the feasibility metrics have been superimposed onto the score plot to help identify regions where physically realistic EMs could be situated, with contours between 0.90 and 0.98 shown with intervals of 0.02 . The outlier to the right of the plot is CM 1.5 MCY 05231 .

\subsubsection{Aqueous alteration grade 1.0-1.2}

Two principle components that accounted for $88 \%$ of the variability were identified and three end members were picked manually to describe the variation in samples with aqueous alteration grades between 1.0 and 1.2. End member 4 (EM4) displays a strong central ridge, with minimal vertical spreading and a peak intensity on the origin. The signal extends along the $\mathrm{B}_{\mathrm{c}}$-axis, to coercivity fields of $300 \mathrm{mT}$, with a pronounced negative signal below the central ridge. This is also characteristic of SD grains, the absence of a negative peak in EM4 compared to EM1 is an artefact of the intensity scaling (Harrison and Lascu, 2014). End member 5 (EM5) displays a tri-lobed pattern similar to vortex state grains, with one lobe extending vertically to $100 \mathrm{mT}$, one lobe extending downwards to $50 \mathrm{mT}$ and a the third extending along the coercivity axis to $150 \mathrm{mT}$. The peak coercivity is off-centre along the $\mathrm{B}_{\mathrm{c}}$-axis at $25 \mathrm{mT}$. End member 6 (EM6) displays a triangular shaped 


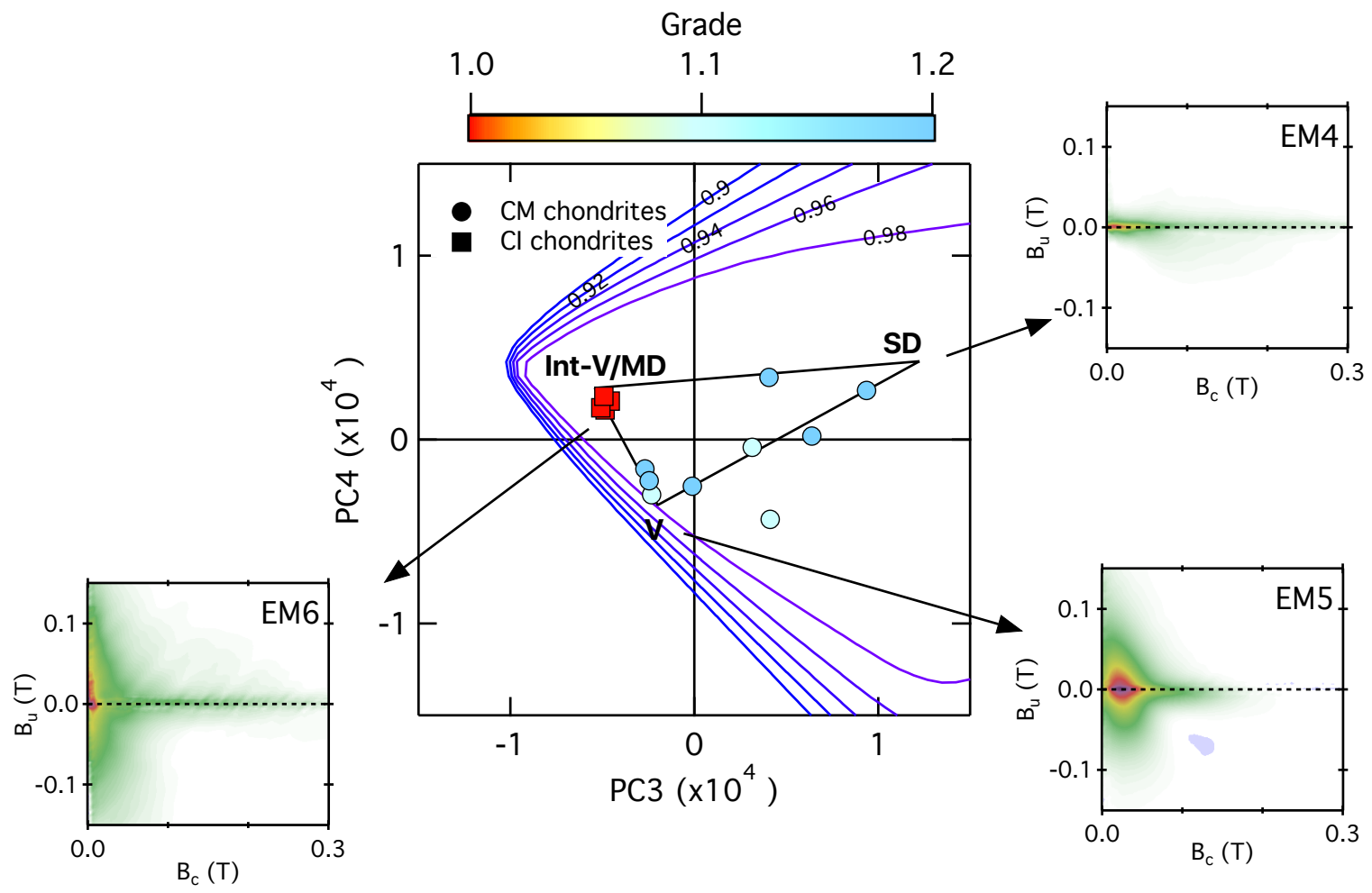

Figure 5: Score plot displaying the proportions of PC3 and PC4 present in each CM and CI chondrite of aqueous alteration grade 1.0-1.2. The positions of the end members are also displayed. EM4 represents non-interacting, SD magnetite; EM5 represents vortex state, magnetite grains; and EM6 represents interacting V/MD grains. The intensity scaling differs between each end member to better highlight their features. Contours of the feasibility metrics have been superimposed onto the score plot to help identify regions where physically realistic EMs could be situated, with contours between 0.90 and 0.98 shown with intervals of 0.02

pattern, resembling interacting $\mathrm{V} / \mathrm{MD}$ grains. There is also a weak signal extending along the $\mathrm{B}_{\mathrm{c}}$-axis. The greater vertical spreading of the signal present in EM6 compared to EM3 could indicate a higher abundance of larger, MD grains in EM6.

CI chondrites plot in distinct principle space, separate from CM1.0-1.2 chondrites. The CI chondrites most closely match the V/MD signal represented by EM6, while the CM1.0-1.2 chondrites plot between the SD and V state EMs, represented by EM4 and EM5, respectively, indicating that they contain a mixture of $\mathrm{SD}$ and vortex state grains.

\section{Discussion}

\subsection{Reason for different magnetite morphologies}

FORC analysis indicates that $\mathrm{CI}$ and $\mathrm{C} 2$-ung chondrites exhibit clearly distinct magnetite morphologies compared to both $\mathrm{CM}$ and $\mathrm{CO}$ chondrites. Magnetite in $\mathrm{CO}$ and $\mathrm{CM}$ chondrites occurs as isolated sub- 

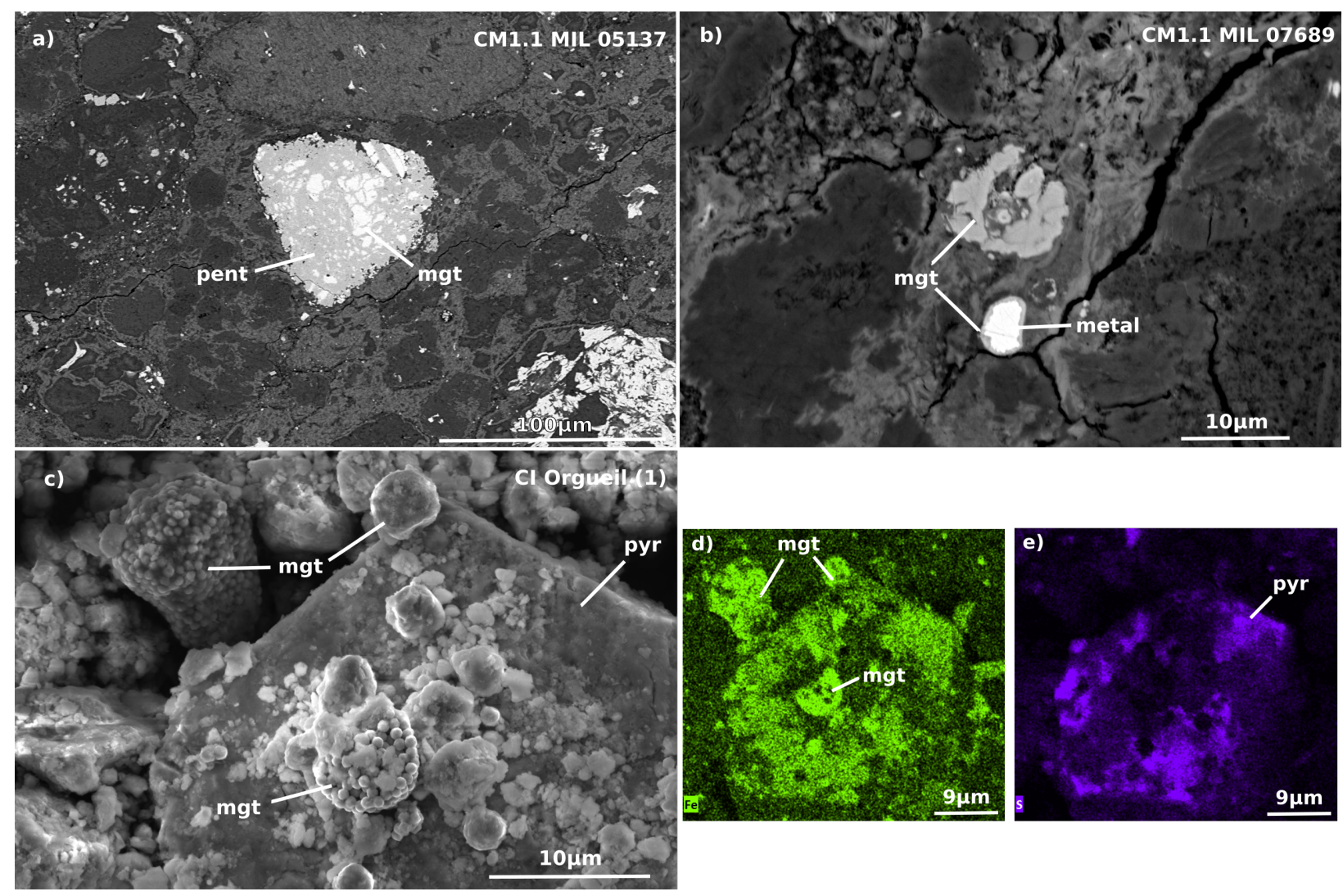

Figure 6: SEM images of selected CM and CI chondrites. a) BSE image of a thin section of the CM1.1 MIL 05137 displaying a magnetite-pentlandite intergrowth as a result of presumed aqueous alteration of a PPI grain, b) BSE image of a thin section of the CM1.1 MIL 07689 showing magnetite forming from oxidation of metal grains, c) SE image of a powder sample of the CI1.0 Orgueil (1) displaying framboids of magnetite and a euhedral, hexagonal grain of pyrrhotite, d) EDS map of the framboids and pyrrhotite in c), displaying the distribution of Fe which is present in both magnetite and pyrrhotite, and e) EDS map of the framboids and pyrrhotite in c), displaying the distribution of S which is only present in pyrrhotite. pent $=$ pentlandite, $\mathrm{mgt}=$ magnetite, pyr $=$ pyrrohtite.

micron to micron scale grains, or as intergrowths in magnetite-pentlandite grains (as seen in Fig. 6a). In contrast, magnetite in CI and C2-ung chondrites occurs as framboids, plaquettes, and micron scale grains. Previous studies have shown that the C2-ung Tagish Lake (TL) shows a very similar triangular-shaped FORC diagram to WIS 91600 and CI chondrites (Bryson et al., 2020a,b), and contains unusual magnetite morphologies including plaquettes and framboids (Greshake et al., 2005). Although these morphologies have been identified in $\mathrm{CM}$ chondrites, they are much rarer and are not the dominant magnetite morphology found in petrographic observations (Hewins et al., 2014). This is also evidenced by the PCA conducted in this study where a few CM chondrites appear to contain a small contribution from the framboid-like endmembers (Figs. 4 and 5). Framboids and plaquettes of magnetite as well as isolated grains of magnetite are both routinely reported in petrographic observations of CR chondrites (Harju et al., 2014). The presence of 
framboids and plaquettes is not due to preservation bias as there is no correlation between their occurrence and the weathering grade of the chondrites examined. Ruling out terrestrial processes, the presence of these unusual forms of magnetite instead of the conventional morphologies of magnetite found in $\mathrm{CO}$ and $\mathrm{CM}$ chondrites (isolated submicron - micron grains) suggests their formation is related to some aspect of the aqueous alteration processes that occurred on different chondrite parent bodies. This suggests that magnetite formation in CI chondrites, Tagish Lake, and WIS 91600 occurred along a different reaction pathway to CM and $\mathrm{CO}$ chondrites, and that a combination of the two pathways may have occurred on the CR parent body.

A number of factors played a role in the aqueous alteration pathway experienced by a chondrite, including: the extent of aqueous alteration; the primitive water:rock ratio of the meteorite; the peak temperature; the starting mineralogy; the accreted metal, sulfides, and silicate phases, and their primitive compositions; the presence of organics; and/or the composition of the fluid. These factors are not independent of each other; for instance, the extent of aqueous alteration is closely linked to the water:rock ratio present on the parent body, and the signature of organics is affected by the temperatures experienced by the chondrite. Each factor has been explored independently in previous studies, and here we review the published observations to investigate their systematic variations in relation to the morphology of magnetite recovered from FORC analysis and petrographic observations with the aim of identifying the controls on the pathway of aqueous alteration.

\subsubsection{Extent of aqueous alteration}

The extent of aqueous alteration experienced by different chondrites is well documented and can be characterised by their vol\% of phyllosilicate (Howard et al., 2015), their petrographic characteristics (Rubin et al., 2007), and their bulk H, C, and N abundances and isotopic compositions (Alexander et al., 2013).

Studies focused on phyllosilicate fraction show that the CI chondrites are essentially fully hydrated and experienced the greatest extent of aqueous alteration among carbonaceous chondrites (King et al., 2015).

Almost all $\mathrm{CM}$ chondrites contain $>50$ vol\% phyllosilicate fraction and exhibit a range of alteration grades, from similar extents to CI chondrites, to mildly altered (Rubin et al., 2007; King et al., 2017), and a few show minimal evidence of alteration (Hewins et al., 2014).

Phyllosilicate fraction studies (Howard et al., 2015) and thermogravimetric analysis (TGA) and IR transmission spectroscopy (Garenne et al., 2014, 2016; Gilmour et al., 2019) indicate that WIS 91600 and Tagish Lake both experienced intermediate extents of aqueous alteration, overlapping with the range experienced by $\mathrm{CM}$ chondrites.

Petrographic observations and bulk H-C-N isotopic studies show that some $\mathrm{CR}$ and most $\mathrm{CO}$ chondrites 
only experienced minimal alteration by fluids (Davidson et al., 2019a,b). The remaining CR chondrites experienced an intermediate range of aqueous alteration extents, spanning petrological grades 2.3-2.8 (Harju et al., 2014) as defined by the scale proposed by Rubin et al. (2007), overlapping with the range experienced by $\mathrm{CM}$ chondrites.

Because abundant magnetite framboids and plaquettes are rarely seen in CM chondrites, despite these meteorites having experienced the overlapping extents of aqueous alteration as some CR chondrites, WIS 91600 and Tagish Lake, this argues that the extent of aqueous alteration does not control the morphology of magnetite.

\subsubsection{Water:rock ratio}

Marrocchi et al. (2018) used the bulk O-isotope compositions of CM, CR, and CO chondrites to estimate their water:rock ratios. These authors calculate ratios of: 0.3-0.4 for CM chondrites; 0.1-0.4 for CR chondrites; and 0.01-0.10 for CO chondrites. CI chondrites have an estimated water:rock ratio of 1.2 (Brearley, 2006). Because CM and CR chondrites have overlapping water:rock ratio ranges, and CR and CI chondrites do not, it is clear that the presence of framboids does not correlate with this parameter.

\subsubsection{Metamorphism}

Differing extents of thermal metamorphism, either during or after aqueous alteration, could affect the thermochemical equilibrium during magnetite formation. This heating can be due to a variety of sources, including the decay of ${ }^{26} \mathrm{Al}$, impact derived heating, and/or solar radiation (Nakamura, 2005; King et al., 2021b). The effect of temperature on magnetite formation has previously been reported in CO chondrites, with progressive thermal alteration (metamorphic grade $\geq 3.2$ ) leading to the destruction of magnetite (Rubin and $\mathrm{Li}, 2019)$. The amount of thermal metamorphism experienced by different chondrites can be examined by a range of methods, including: Cr concentration of FeO-rich chondrule olivine (Davidson et al., $2019 \mathrm{~b}){ }^{18} \mathrm{O}-$ isotope fractionation (Jilly-Rehak et al., 2018); modifications of organics (Alexander et al., 2013); structural order of polyaromatic carbonaceous material (Bonal et al., 2016); identification of characteristic mineral phases and thermally induced mineralogical changes (King et al., 2021b); and the Co/Ni value of metal grains (Kimura et al., 2011).

CO 3.0-3.1 chondrites underwent minimal aqueous alteration and thermal metamorphism (not experiencing temperatures $>100-300^{\circ} \mathrm{C}$, indicated by the presence of fayalite), and contain $\sim 2-8$ vol $\%$ magnetite. CO 3.2-3.6 chondrites experienced peak temperatures up to $\sim 600^{\circ} \mathrm{C}$ and show much lower magnetite abundances, due to the reduction of magnetite to form fayalite and secondary kamacite (Rubin and Li, 2019). The specific CO chondrites examined in this study are among the most primitive, with DOM 08006 experi- 
encing similar, or lower temperatures of alteration compared to CR chondrites, and MIL 090010 experiencing marginally higher temperatures (Bonal et al., 2016; Davidson et al., 2019a; Rubin and Li, 2019).

Using ${ }^{18} \mathrm{O}$-isotope fractionation, aqueous alteration on the $\mathrm{CR}$ parent body has been argued to have been a low-temperature process, occurring at $55-88^{\circ} \mathrm{C}$ (Jilly-Rehak et al., 2018). Measurements of the Cr composition in FeO-rich olivine, and modification of organics support this finding (Alexander et al., 2013; Davidson et al., 2019b), indicating that CR chondrites underwent thermal alteration at much lower temperatures compared to the $\mathrm{CI}$ chondrites, which experienced maximum temperatures of $\sim 150^{\circ} \mathrm{C}$ (King et al., 2015).

$\mathrm{CM}$ chondrites underwent aqueous alteration at temperatures of $<150-300^{\circ} \mathrm{C}$, and some appear to have experienced peak metamorphic temperatures of $\sim 200$ to $>700^{\circ} \mathrm{C}$ due to transient impact heating events that do not appear to have a measurable effect on magnetite morphology (King et al., 2021b; Suttle et al., 2021).

Investigation of the insoluble organic matter (IOM) and conditions inferred by experiments indicates that Tagish Lake experienced aqueous alteration at temperatures between $<150-300^{\circ} \mathrm{C}$, comparable to $\mathrm{CM}$ chondrites (Herd et al., 2011). The IOM and the structure of organics present in WIS 91600 indicate that it experienced short-duration thermal metamorphism at temperatures between $400-500^{\circ} \mathrm{C}$ (Yabuta et al., 2010; Quirico et al., 2018), which is unlikely to have affected the morphology of magnetite.

Due to the observations that: (1) CR and CI chondrites contain magnetite framboids and plaquettes, despite having experienced different extents of metamorphism; and (2) $\mathrm{CO}$ and $\mathrm{CM}$ chondrites do not contain magnetite framboids and plaquettes despite having undergone alteration at temperatures similar to CR chondrites, WIS 91600, and Tagish Lake, the extent of thermal metamorphism does not appear to control magnetite morphology in a systematic way.

It is possible that subsequent heating during laboratory analysis could have led to the formation, or destruction, of magnetite framboids and plaquettes. However, FORC diagrams of laboratory heated and unheated samples of the CM Murchison and Orgueil and Ivuna do not exhibit significant variations, indicating that the morphology and characteristics of the magnetite have not been altered by laboratory heating (Fig. S4-6).

\subsubsection{Presence of organics}

Organic matter is present in the chondrites in solvent soluble (SOM) and IOM forms. These are thought to form at very low temperatures in the presolar molecular cloud and/or interstellar environments, and have undergone subsequent thermal and aqueous processing on chondrite parent bodies (Alexander et al., 2017). 

or iron sulfides, following equations 2 and 3 .

$$
\text { Metal }+ \text { Water } \rightleftharpoons \text { Magnetite }+H_{2}
$$

$$
\text { Troilite }+ \text { Water } \rightleftharpoons \text { Magnetite }+\mathrm{H}_{2} \mathrm{~S}+\mathrm{H}_{2}
$$
impact the morphology of magnetite. magnetite morphology.

\subsubsection{Starting mineralogy}

In situ studies of carbonaceous chondrites indicate close association of IOM with phyllosilicates in Tagish Lake and Orgueil (Herd et al., 2011; Alexander et al., 2017). This suggests that they are associated with the process of aqueous alteration in chondrites, possibly affecting the composition of the fluid, which could

The bulk concentration of $\mathrm{H}, \mathrm{N}$, and $\mathrm{C}$ of IOM in $\mathrm{CI}, \mathrm{CM}, \mathrm{CO}$, and $\mathrm{CR}$ chondrites show no systematic variation with the morphology of magnetite, arguing that organic abundance and the nature of the organics present during aqueous alteration does not control magnetite morphology (Alexander et al., 2017, 2018b). In particular, $\mathrm{CO}$ and $\mathrm{CR}$ chondrites contain similar bulk $\mathrm{C}$ concentrations, and the IOM contents of CI, $\mathrm{CM}$ and $\mathrm{CR}$ chondrites are similar, demonstrating the lack of correlation between organic presence and

Petrographic observations of primitive CO chondrites (e.g., DOM 08006) and CR chondrites (e.g., MIL 090657) find minimal evidence of aqueous alteration. Despite this, the least altered chondrites from both groups contain abundant magnetite (Davidson et al., 2019a,b). This observation suggests that magnetite must have been one of the first phases to form during aqueous alteration, before significant phyllosilicate formation or other signatures of alteration. Magnetite can be formed from alteration of either metal grains

7

The precursor mineralogy that undergoes alteration could control which morphology of magnetite is subsequently formed.

$C M$ and $C O$ chondrites: In $\mathrm{CM}$ and $\mathrm{CO}$ chondrites, metal grains are often the first phases to oxidise on hydration; this is evidenced by the CO chondrites DOM 08006 and MIL 090010, which contain $\leq 2$ vol\% phyllosilicate but have magnetite abundances that exceed those of any CM chondrites (Alexander et al., 2018a). This magnetite formed from metal during limited amounts of aqueous alteration (Rubin and Li, 2019). Alteration from metal to magnetite is further evidenced by SEM images of CM and CO chondrites which clearly display magnetite mantling metal grains (Fig. 6) (Palmer and Lauretta, 2011; Davidson et al., 2019a; Rubin and Li, 2019). Magnetite can also form through the alteration of pyrrhotite in pyrrhotite- 
pentlandite intergrowth (PPI) grains in the CM chondrites (Fig. 6) (Singerling and Brearley, 2020). In all cases, the magnetite formed rarely adopts the framboids and plaquettes seen in the CI, CR, and C2-ung chondrites.

CI and C2-ung chondrites: Framboidal magnetite and magnetite plaquettes form from alteration of sulphides in CI chondrites. This is seen in BSE images where magnetite is shown to precipitate in the space left behind from the dissolution of the sulfide grains and the large-scale morphology of the pyrrhotite is retained. The phenomenon of framboid and plaquette magnetite forming in the place of sulfide grains is seen in the ungrouped C2 chondrite Tagish Lake (Greshake et al., 2005). Close association of framboidal magnetite with sulfides has also been reported in WIS 91600 (Brearley, 2004). Though no primary metal abundances in the CI and C2-ung chondrites have been reported (King et al., 2015), petrographic observations indicate that large metal grains may alter to serpentine through a series of currently unknown intermediary phases during aqueous alteration (Brearley, 2004).

$C R$ chondrites: In CR chondrites, magnetite forms from alteration of both sulphides, to form framboids and plaquettes, and metal grains, to form single grains (Harju et al., 2014; Singerling and Brearley, 2020; Schrader et al., 2021).

The composition of the starting mineralogy could affect the pathways of aqueous alteration. In pristine $\mathrm{CO}$ and $\mathrm{CR}$ chondrites, the composition of Fe-Ni metal and Fe-sulfides is not significantly different (Davidson et al., 2019a,b), and the variation in measured Fe-sulfide composition in the CM, CI, and CR chondrites appears to be a product of extent of aqueous alteration (Harju et al., 2014; Singerling and Brearley, 2018). Because the compositions of the initial metal and Fe-sulfides present in the CO, CM, CI, and CR chondrites do not significantly differ between the chondrite groups, initial compositional variation among these minerals cannot account for the differences in alteration pathways.

Based on the petrographic and magnetic measurements, it is clear that metal grains were present in the starting mineralogy of the $\mathrm{CM}, \mathrm{CO}, \mathrm{CR}$, and $\mathrm{CI}$ chondrite groups and although metal can readily alter with water (as seen in $\mathrm{CO}$ chondrites), it only alters to form magnetite in the $\mathrm{CM}, \mathrm{CO}$, and $\mathrm{CR}$ chondrites. Sulfides with similar primitive compositions were also present in the starting mineralogy of all chondrite groups examined here, however, only in the CI, C2-ung, and CR chondrites do they alter to form magnetite framboids and plaquettes. The precursor mineralogy relates to the morphology of magnetite formed during aqueous alteration (metal transforms to single magnetite grains in $\mathrm{CM}, \mathrm{CO}$, and $\mathrm{CR}$ chondrites, and sulfides transform to framboid and plaquette magnetite in the CI, C2-ung, and CR chondrites), however, only one pathway of alteration occurs in each group (except the CR chondrites) despite the presence of the necessary 
starting minerals for both pathways to have occurred. As such, because all groups have the potential for both pathways to have occurred, the initial presence or absence of metal or sulfide grains is not the reason for the differences in the observed end product. In this sense, the starting mineralogy does not control the morphology of magnetite formed during aqueous alteration.

\subsubsection{Fluid composition}

The composition of the hydrous fluid within chondrite parent bodies could have potentially affected the aqueous reactions that occurred on these asteroids. This composition is expected to have evolved as different phases reacted, such that the initial fluid composition will have been controlled by the composition of the ice accreted into the parent asteroid of each group. Because magnetite was one of the very first phases to form during chondrite aqueous alteration (Rubin and Li, 2019; Davidson et al., 2019a,b), the composition of the fluid from which this mineral formed will have been governed predominantly by that of the ice accreted into its parent body rather than an evolved fluid composition (Brearley, 2006). Because we are able to systematically rule out variations in the other major factors (discussed in Sections 4.1.1 to 4.1.5) as the driving force behind the morphology of magnetite, we argue that the composition of the ice accreted into the parent bodies of Tagish Lake, WIS 91600, CI chondrites, and CR chondrites was different to that incorporated into the $\mathrm{CO}$ and $\mathrm{CM}$ parent asteroids.

Our current understanding of the variation of ice composition in the protoplanetary disk is not well consolidated. However, Collings et al. (2004) found that after water ice, the next molecule that is expected to have condensed out of the solar nebula in geochemically relevant quantities is ammonia. This molecule has been found on Ceres in the form of ammoniated silicates (Ehlmann et al., 2018), on Pluto's moon, Charon, as ammonia hydrates (DeMeo et al., 2014), and on comet 67P as ammonium salts (Poch et al., 2020). High yields of ammonia have also been identified in the IOM within CR and CI chondrites, attesting to its presence in these groups (Pizzarello and Williams, 2012).

The accretion of ammoniated ice into some chondrite parent bodies could have led to their primitive fluids being more alkaline. The presence of this basic fluid may then have affected the pathway of aqueous alteration observed among some carbonaceous chondrites, possibly influencing which minerals formed and the morphologies they adopted. Circumstantial evidence for this includes the possible influence of alkaline fluids on the morphology of magnetite highlighted by White et al. (2020), which indicates that framboids of magnetite formed under basic conditions. These authors use atom probe tomography to image the surface of framboidal magnetite in Tagish Lake, identifying the presence of $\mathrm{Na}$ and $\mathrm{Mg}$ cations, which provide an overall zero static surface charge on the individual magnetite grains only possible in more basic fluids. The 
presence of these surface cations within this fluid prevents smaller magnetite grains from coagulating to form a larger grain, enabling well-ordered framboids of magnetite to form. Also identified by this study was Na clustered on subgrain boundaries trapped within magnetite framboids, implying an excess of sodium in the parental fluid that led to more alkaline compositions. Much of the potential ammonia originally accreted into chondrites is unlikely to be measured in laboratory studies of these samples at the present day because it readily sublimates from its principal hosts (ammonium salts and saponite) at room temperature, evidenced by the lack of ammonia observed in the coma dust grains from comet 67P analysed on the Rosetta spacecraft (Poch et al., 2020).

Ammonia is predicted to condense out of the solar nebula at lower temperatures ( $90 \mathrm{~K})$ than water ice ( 160 K) (Collings et al., 2004; Dodson-Robinson et al., 2009), and so would be present in its solid form in cooler regions of the disk or at later times as the disk cooled. As such, the reliable identification of ammoniated ice in some chondrites could place constraints on the heliocentric distances and/or timings at which their parent asteroids accreted (see Fig. 7). Coupled with previous studies that indicate that: (1) the CI chondrites accreted and underwent aqueous alteration at similar times as the $\mathrm{CM}$ and $\mathrm{CO}$ chondrites (approximately 3.1-4.1 Ma, 3.0-4.2 Ma, and 2.5-2.9 Ma after CAI formation, respectively), and could represent the most distal carbonaceous chondrite group (>15 AU) (Desch et al., 2018; Pravdivtseva et al., 2018); (2) the Tagish Lake and WIS 91600 parent asteroids appear to have accreted at greater distances (>8-13 $\mathrm{AU}$ and $\sim 10 \mathrm{AU}$, respectively) compared to the $\mathrm{CM}, \mathrm{CO}$ and $\mathrm{CR}$ chondrites ( 3-4 AU) (Desch et al., 2018; Bryson et al., 2020a,b); and (3) the CR chondrite parent body accreted 0.1-1.5 Ma later than CI, CO, and CM parent bodies (Schrader et al., 2017; Desch et al., 2018), we hypothesise that our observation of abundant framboidal and plaquette magnetite could imply that the Tagish Lake, WIS 91600, $\mathrm{CR}$, and CI chondrite parent bodies formed at more distal locations or younger times than those of the CM and $\mathrm{CO}$ parent bodies. The younger recovered accretion age of the $\mathrm{CR}$ chondrite parent body compared to the more distal CI chondrites indicates that the potential inward migration of the ammonia ice line as the protoplanetary disk cooled could explain the presence of ammonia in the CR chondrites.

The accretion of ammoniated ice in the most distal chondrites could impact the nitrogen budget of small planetary bodies and has consequent implications for volatile delivery to planetesimals in the early solar system.

If different ice composition is the controlling factor on magnetite morphology, this can then provide an explanation for the presence of rare magnetite framboids and plaquettes in some $\mathrm{CM}$ chondrites (Hewins et al., 2014). The water composition during the alteration of these meteorites could have been affected 
by its local mineralogy, allowing for pockets of more alkaline fluid that potentially led to the formation of limited framboidal magnetite, without the need for accretion of ammoniated ice (Palmer and Lauretta, 2011). Moreover, links between alkaline fluids and magnetite morphology could be explored in future laboratory experiments in an effort to better understand how the properties of magnetite vary with fluid compositions and the consequences of this on our understanding of chondrite alteration histories and parent body formation locations.

\subsection{Tagish Lake: A case study}

Tagish Lake provides a unique opportunity to investigate which factors could control the generation of different magnetite morphologies. The different stones in the Tagish Lake fall are heterogeneous, with each stone exhibiting different water:rock ratios, and having experienced varying extents of thermal and aqueous alteration. For instance, stone TL5b has been found to have altered at $<50^{\circ} \mathrm{C}$ (Blinova et al., 2014) while the rest of the Tagish Lake protolith has been found to have altered at $<150^{\circ} \mathrm{C}$ (Herd et al., 2011). The stones also represent different stages of hydrous alteration, i.e., TL5b (the least altered) $<$ TL11h $<$ TL11i (the most altered). Despite these variations, all of the TL stones contain abundant framboids and plaquettes of magnetite (Blinova et al., 2014).

As outlined previously, but now identified in a singular meteorite, differences in water:rock ratios and the extent of aqueous and thermal alteration do not control the morphology of magnetite and the pathway of aqueous alteration. These properties can vary depending on the location of the stones within the parent body, as different depths within the parent body experienced different temperatures and amounts of aqueous alteration. The controlling factor on the pathway of aqueous alteration must instead be an independent property that is constant throughout the Tagish Lake parent body, such as the composition of the accreted ice. Upon melting, this ice controls the chemistry of the aqueous fluid and therefore the morphology of magnetite formed.

\section{Conclusions}

1. Using bulk magnetic characterisation techniques, we find that magnetite grain size and morphology varies systematically between CM, CO, CI and C2-ung chondrites.

2. Estimated modal abundances from bulk magnetic measurements suggest that there is no correlation between the extent of aqueous alteration and the amount of magnetite formed in CM chondrites.

3. The magnetic signal in $\mathrm{CM}$ chondrites is dominated by $<0.1 \mu \mathrm{m} \mathrm{SD}$ magnetite in the groundmass, formed from alteration of Fe-Ni metal. 
a)

\section{$\mathrm{CO}, \mathrm{CM} \& \mathrm{CR}$ chondrites}

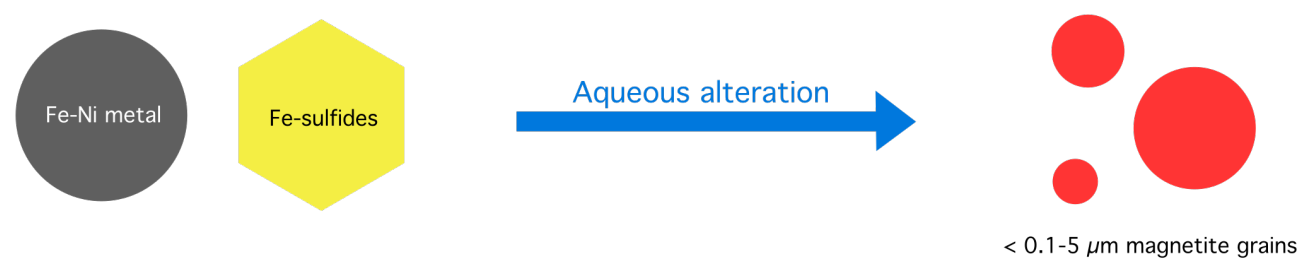

b)

\section{$\mathrm{CR}, \mathrm{Cl} \& \mathrm{C} 2$ chondrites}
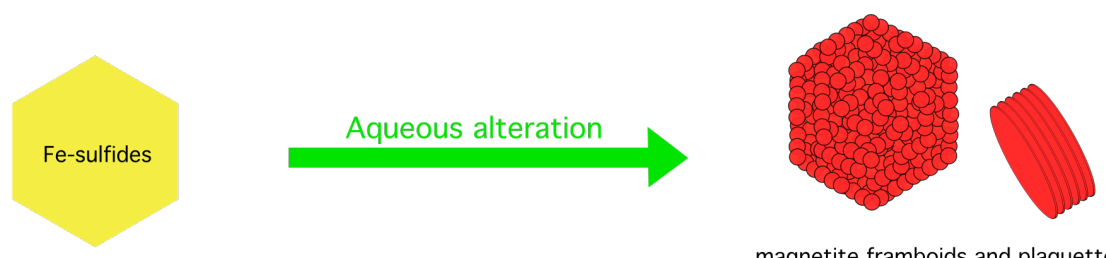

magnetite framboids and plaquettes

c)
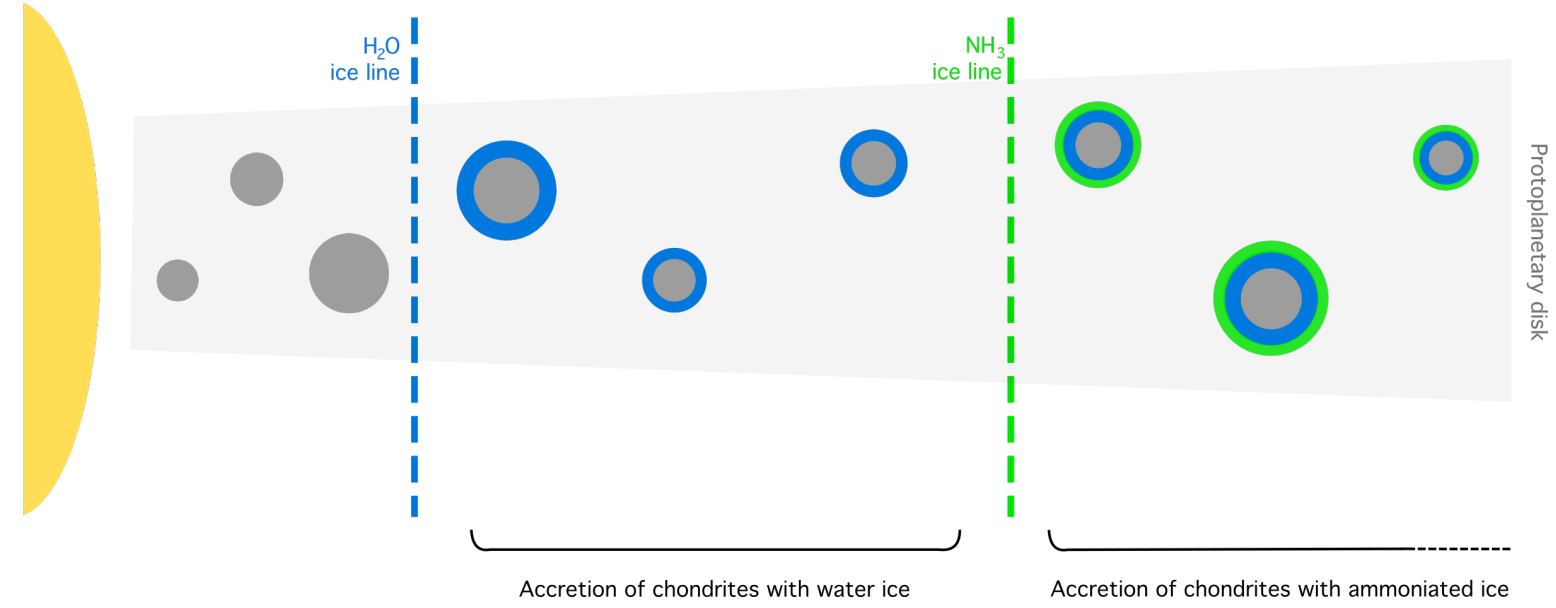

Figure 7: a) Generalised reaction pathway of metal grains and Fe-sulfides within the CO, CM and CR chondrites during aqueous alteration, where $<0.1-5 \mathrm{\mu m}$ magnetite grains are typically formed, b) Generalised reaction pathway of Fe-sulfides within the CR, CI, and C2-ung chondrites during aqueous alteration, where framboids and plaquettes of magnetite are formed due to alkaline conditions during aqueous alteration, c) Schematic figure illustrating the location of accretion of the carbonaceous chondrites, proposed in Section 4.2.6. The CO and CM chondrites accrete past the water ice line and the CI chondrites, WIS 91600, and Tagish Lake accrete at greater radial distances, past the ammonia ice line. The accretion of ammoniated ice (in green) changes the fluid composition of the chondrite parent body, leading to alkaline conditions during aqueous alteration, facilitating the formation of framboids and plaquettes of magnetite.

4. Unusual morphologies of magnetite, including framboids and plaquettes, are found in CI, CR, and C2-ung chondrites, including Tagish Lake and WIS 91600. This magnetite was formed during a different pathway of aqueous alteration compared to $\mathrm{CM}$ and $\mathrm{CO}$ chondrites, which lack these magnetite morphologies in significant abundances. 
5. Ruling out systemic variations in the extent of aqueous alteration, the primitive water:rock ratio of the meteorite, the degree of metamorphism, the primitive mineralogy and its composition, and the presence of organics, we propose that the formation of unusual magnetite morphologies is controlled by the composition of ice accreted into the different parent asteroids. The most feasible way by which the composition of the ice changed is through the condensation of ammonia from the protoplanetary disk. This process would have lead to more alkaline fluids upon melting on their parent bodies, which could have caused alteration to progress along fundamentally different pathways.

6. Given constraints on parent body accretion ages, this argues that compared to CM and CO chondrites, the CI chondrites, Tagish Lake and WIS 91600 originate from a more distal region beyond the ammonia ice line, and that the ice line could have migrated inwards by the comparatively young time of accretion of the CR chondrite parent body.

7. The volatile nature of many ammoniated compounds suggests that a considerable portion of this molecule will have been lost from CI, CR, and C2-ung chondrites on Earth. However, our results hint that the pre-terrestrial $\mathrm{N}$ and $\mathrm{H}$ budgets of these chondrites may have been significantly higher than those measured today.

\section{Acknowledgements}

The authors would like to thank Dr Iris Buisman for her help with the SEM, and Dr Oliver Shorttle and Professor Nicholas Tosca for the insightful discussions. We would also like to thank the two anonymous reviewers for their constructive comments which have improved the clarity and quality of the manuscript. The authors would also like to thank the Natural History Museum in the UK, and the Antarctic Search for Meteorites (ANSMET) program (which has been funded by NSF and NASA and curated by the Department of Mineral Sciences of the Smithsonian Institution and Astromaterials Curation Office at NASA Johnson Space Center) for use of their meteorite samples. The magnetic data presented in this paper can be found on the MagIC database, with the DOI:10.7288/V4/MAGIC/19215 (https://www2.earthref.org/ MagIC). 


\section{References}

Alexander, C., Cody, G., De Gregorio, B., Nittler, L., Stroud, R., 2017. The nature, origin and modification of insoluble organic matter in chondrites, the major source of Earth's C and N. Geochemistry 77, 227-256. doi:10.1016/j.chemer.2017.01.007.

Alexander, C., Greenwood, R., Bowden, R., Gibson, J., Howard, K., Franchi, I., 2018a. A multi-technique search for the most primitive CO chondrites. Geochimica et Cosmochimica Acta 221, 406-420. doi:10.1016/j.gca.2017.04.021.

Alexander, C., Howard, K., Bowden, R., Fogel, M., 2013. The classification of CM and CR chondrites using bulk H, C and N abundances and isotopic compositions. Geochimica et Cosmochimica Acta 123, 244-260. doi:10.1016/j.gca.2013.05.019.

Alexander, C., McKeegan, K., Altwegg, K., 2018b. Water Reservoirs in Small Planetary Bodies: Meteorites, Asteroids, and Comets. Space Science Reviews 214. doi:10.1007/s11214-018-0474-9.

Bates, H., King, A., Donaldson Hanna, K., Bowles, N., Russell, S., 2020. Linking mineralogy and spectroscopy of highly aqueously altered CM and CI carbonaceous chondrites in preparation for primitive asteroid sample return. Meteoritics \& Planetary Science doi:10.1111/maps.13411.

Blinova, A., Zega, T., Stroud, R., 2014. Testing variations within the Tagish Lake meteorite-I: Mineralogy and petrology of pristine samples. Meteoritics \& Planetary Science 49, 473-502. doi:10.1111/maps.12271.

Bonal, L., Quirico, E., Flandinet, L., Montagnac, G., 2016. Thermal history of type 3 chondrites from the Antarctic meteorite collection determined by Raman spectroscopy of their polyaromatic carbonaceous matter. Geochimica et Cosmochimica Acta 189, 312-337. doi:10.1016/j.gca.2016.06.017.

Brearley, A., 2004. A unique style of alteration of iron-nickel metal in WIS91600, an unusual C2 carbonaceous chondrite. 35th Lunar and Planetary Science Conference.

Brearley, A., 2006. The Action of Water, in: Meteorites and the Early Solar System II. University of Arizona Press, pp. $584-624$.

Bryson, J., Weiss, B., Biersteker, J., King, A., Russell, S., 2020a. Constraints on the Distances and Timescales of Solid Migration in the Early Solar System from Meteorite Magnetism. The Astrophysical Journal 896. doi:10.3847/1538-4357/ab91ab.

Bryson, J., Weiss, B., Lima, E., Gattacceca, J., Cassata, W., 2020b. Evidence for Asteroid Scattering and Distal Solar System Solids From Meteorite Paleomagnetism. The Astrophysical Journal 892. doi:10.3847/1538-4357/ab7cd4.

Collings, M., Anderson, M., Chen, R., Dever, J., Viti, S., Williams, D., McCoustra, M., 2004. A laboratory survey of the thermal desorption of astrophysically relevant molecules. Monthly Notices of the Royal Astronomical Society 354, 11331140. doi:10.1111/j.1365-2966.2004.08272.x.

Cournède, C., Gattacceca, J., Gounelle, M., Rochette, P., Weiss, B., Zanda, B., 2015. An early solar system magnetic field recorded in CM chondrites. Earth and Planetary Science Letters 410, 62-74.

Davidson, J., Alexander, C., Stroud, R., Busemann, H., Nittler, L., 2019a. Mineralogy and petrology of Dominion Range 08006: A very primitive CO3 carbonaceous chondrite. Geochimica et Cosmochimica Acta 265, $259-278$. doi:10.1016/j.gca.2019.08.032

Davidson, J., Schrader, D., Alexander, C., Nittler, L., Bowden, R., 2019b. Re-examining thermal metamorphism of the Renazzo-like (CR) carbonaceous chondrites: Insights from pristine Miller Range 090657 and shock-heated Graves Nunataks 06100. Geochimica et Cosmochimica Acta 267, 240-256. doi:10.1016/j.gca.2019.09.033.

DeMeo, F., Dumas, C., Cook, J., Carry, B., Merlin, F., Verbiscer, A., Binzel, R., 2014. Spectral variability of Charon's 2.21-lm feature. Icarus 246, 213-219. doi:10.1016/j.icarus.2014.04.010.

Desch, S., Kalyaan, A., Alexander, C., 2018. The Effect of Jupiter's Formation on the Distribution of Refractory Elements and Inclusions in Meteorites. The Astrophysical Journal Supplement Series 238. doi:10.3847/1538-4365/aad95f.

Dodson-Robinson, S., Willacy, K., Bodenheimer, P., Turner, N., Beichman, C., 2009. Ice lines, planetesimal composition and solid surface density in the solar nebula. Icarus 200, 672-693. doi:10.1016/j.icarus.2008.11.023.

Egli, R., 2013. VARIFORC: An optimized protocol for calculating non-regular first-order reversal curve (FORC) diagrams. Global and Planetary Change 110, 302-320. doi:10.1016/j.gloplacha.2013.08.003.

Ehlmann, B., Hodyss, R., Bristow, T., Rossman, G., Ammannito, E., De Sanctis, M., Raymond, C., 2018. Ambient and cold-temperature infrared spectra and XRD patterns of ammoniated phyllosilicates and carbonaceous chondrite meteorites relevant to Ceres and other solar system bodies. Meteoritics \& Planetary Science 53, 1884-1901. doi:10.1111/maps.13103.

Garenne, A., Beck, P., Montes-Hernandez, G., Brissaud, O., Schmitt, B., Quirico, E., Bonal, L., Beck, C., Howard, K., 2016. Bidirectional reflectance spectroscopy of carbonaceous chondrites: Implications for water quantification and primary composition. Icarus 264, 172-183. doi:10.1016/j.icarus.2015.09.005.

Garenne, A., Beck, P., Montes-Hernandez, G., Chiriac, R., Toche, F., Quirico, E., Bonal, L., Schmitt, B., 2014. The abundance and stability of "water" in type 1 and 2 carbonaceous chondrites (CI, CM and CR). Geochimica et Cosmochimica Acta 137, 93-112. doi:10.1016/j.gca.2014.03.034

Gilmour, C., Herd, C., Beck, P., 2019. Water abundance in the Tagish Lake meteorite from TGA and IR spectroscopy: Evaluation of aqueous alteration. Meteoritics \& Planetary Science 54, 1951-1972. doi:10.1111/maps.13362.

Greshake, A., Krot, A., Flynn, G., Keil, K., 2005. Fine-grained dust rims in the Tagish Lake carbonaceous chondrite: Evidence for parent body alteration. Meteoritics \& Planetary Science 40, 1413-1431. doi:10.1111/j.1945-5100.2005.tb00410.x.

Harju, E., Rubin, A., Ahn, I., Choi, B., Ziegler, K., Wasson, J., 2014. Progressive aqueous alteration of CR carbonaceous chondrites. Geochimica et Cosmochimica Acta 139, 267-292. doi:10.1016/j.gca.2014.04.048.

Harrison, R., Feinberg, J., 2008. FORCinel: An improved algorithm for calculating first-order reversal curve distributions using locally weighted regression smoothing. Geochemistry Geophysics Geosystems 9. doi:10.1029/2008GC001987.

Harrison, R., Lascu, I., 2014. FORCulator: A micromagnetic tool for simulating first-order reversal curve diagrams. Geochemistry Geophysics Geosystems 15, 4671-4691. doi:10.1002/2014GC005582.

Harrison, R., Muraszko, J., Heslop, D., Lascu, I., Muxworthy, A., Roberts, A., 2018. An Improved Algorithm for Unmixing 
First-Order Reversal Curve Diagrams Using Principal Component Analysis. Geochemistry Geophysics Geosystems 19, 1595-1610. doi:10.1029/2018GC007511.

Harrison, R., Zhao, X., Hu, P., Sato, T., Heslop, D., Muxworthy, A., Oda, H., Kuppili, V., Roberts, A., 2019. Simulation of remanent, transient, and induced first-order reversal curve (FORC) diagrams for interacting particles with uniaxial, cubic, and hexagonal anisotropy. Journal of Geophysical Research: Solid Earth 124, 404-429. doi:10.1029/2019JB018050.

Herd, C., Blinova, A., Simkus, D., Huang, Y., Tarozo, R., Alexander, C., Gyngard, F., Nittler, L., Cody, G., Fogel, M., Kebukawa, Y., Kilcoyne, A., Hilts, R., Slater, G., Glavin, D., Dworkin, J., Callahan, M., Elsila, J., De Gregorio, B., Stroud, R., 2011. Origin and Evolution of Prebiotic Organic Matter As Inferred from the Tagish Lake Meteorite. Science 331, 1304-1307. doi:10.1126/science.1203290.

Hewins, R., Bourot-Denise, M., Zanda, B., Leroux, H., Barrat, J., Humayun, M., Gopel, C., Greenwood, R., Franchi, I., Pont, S., Lorand, J., Cournede, C., Gattacceca, J., Rochette, P., Kuga, M., Marrocchi, Y., Marty, B., 2014. The Paris meteorite, the least altered CM chondrite so far. Geochimica et Cosmochimica Acta 124, 190-222. doi:10.1016/j.gca.2013.09.014.

Howard, K., Alexander, C., Schrader, D., Dyl, K., 2015. Classification of hydrous meteorites (CR, CM and C2 ungrouped) by phyllosilicate fraction: PSD-XRD modal mineralogy and planetesimal environments. Geochimica et Cosmochimica Acta 149, 206-222. doi:10.1016/j.gca.2014.10.025.

Jilly-Rehak, C., Huss, G., Nagashima, K., Schrader, D., 2018. Low-temperature aqueous alteration on the CR chondrite parent body: Implications from in situ oxygen-isotope analyses. Geochimica et Cosmochimica Acta 222 , $230-252$. doi:10.1016/j.gca.2017.10.007.

Kerridge, J.F., Mackay, A.L., Boynton, W.V., 1979. Magnetite in CI Carbonaceous Meteorites: Origin by Aqueous Activity on a Planetesimal Surface. Science 205, 395-397. doi:10.1126/science.205.4404.395.

Kimura, M., Grossman, J., Weisberg, M., 2011. Fe-Ni metal and sulfide minerals in CM chondrites: An indicator for thermal history. Meteoritics \& Planetary Science 46, 431-442. doi:10.1111/j.1945-5100.2010.01164.x.

King, A., Mason, E., Bates, H., Schofield, P., Donaldson Hanna, K., Bowles, N., Russell, S., 2021a. Tracing the earliest stages of hydrothermal alteration on the CM chondrite parent body. Meteoritics \& Planetary Science. Meteoritics \& Planetary Science doi:(in press).

King, A., Russell, S., Schofield, P., Humphreys-Williams, E., Strekopytov, S., Abernethy, F., Verchovsky, B., Grady, M., 2019. The alteration history of the Jbilet Winselwan CM carbonaceous chondrite: An analog for C-type asteroid sample return. Meteoritics \& Planetary Science 54, 521-543. doi:10.1111/maps.13224.

King, A., Schofield, P., Howard, K., Russell, S., 2015. Modal mineralogy of CI and CI-like chondrites by X-ray diffraction. Geochimica et Cosmochimica Acta 165, 148-160. doi:10.1016/j.gca.2015.05.038.

King, A., Schofield, P., Russell, S., 2017. Type 1 aqueous alteration in CM carbonaceous chondrites: Implications for the evolution of water-rich asteroids. Meteoritics \& Planetary Science 52, 1197-1215. doi:10.1111/maps.12872.

King, A., Schofield, P., Russell, S., 2021b. Thermal alteration of CM carbonaceous chondrites: Mineralogical changes and metamorphic temperatures. Geochimica et Cosmochimica Acta 298, 167-190. doi:10.1016/j.gca.2021.02.011.

Lee, M., Cohen, B., King, A., Greenwood, R., 2019. The diversity of CM carbonaceous chondrite parent bodies explored using Lewis Cliff 85311. Geochimica et Cosmochimica Acta 264, 224-244. doi:10.1016/j.gca.2019.07.027.

Macke, R., Consolmagno, G., Britt, D., 2011. Density, porosity, and magnetic susceptibility of carbonaceous chondrites. Meteoritics \& Planetary Science 46, 1842-1862. doi:10.1111/j.1945-5100.2011.01298.x.

Marrocchi, Y., Bekaert, D., Piani, L., 2018. Origin and abundance of water in carbonaceous asteroids. Earth and Planetary Science Letters 482, 23-32. doi:10.1016/j.epsl.2017.10.060.

Nakamura, T., 2005. Post-hydration thermal metamorphism of carbonaceous chondrites. Journal of Mineralogical and Petrological Sciences 100, 260-272. doi:10.2465/jmps.100.260.

Palmer, E., Lauretta, D., 2011. Aqueous alteration of kamacite in CM chondrites. Meteoritics \& Planetary Science 46, 1587-1607. doi:10.1111/j.1945-5100.2011.01251.x.

Pizzarello, S., Williams, L.B., 2012. Ammonia in the early solar system: An account from carbonaceous meteorites. The Astrophysical Journal 749, 161. doi:10.1088/0004-637X/749/2/161.

Poch, O., Istiqoman, I., Quirico, E., Beck, P., Schmitt, B., Theulé, P., Faure, A., Hily-Blant, P., Bonal, L., Raponi, A., Ciarniello, M., Rousseau, B., Potin, S., Brissaud, O., Flandinet, L., Filacchione, G., Pommerol, A., Thomas, N., Kappel, D., Mennella, V., Moroz, L., Vinogradoff, V., Arnold, G., Erard, S., Bockelée-Morvan, D., Leyrat, C., Capaccioni, F., Sanctis, M., Longobardo, A., Mancarella, F., Palomba, E., Tosi, F., 2020. Ammonium salts are a reservoir of nitrogen on a cometary nucleus and possibly on some asteroids 367. doi:10.1126/science.aaw7462.

Pravdivtseva, O., Krot, A., Hohenberg, C., 2018. I-Xe dating of aqueous alteration in the CI chondrite Orgueil: I. Magnetite and ferromagnetic separates. Geochimica et Cosmochimica Acta 227, 38-47. doi:10.1016/j.gca.2018.02.004.

Quirico, E., Bonal, L., Beck, P., Alexander, C., Yabuta, H., Nakamura, T., Nakato, A., Flandinet, L., Montagnac, G., SchmittKopplin, P., Herd, C., 2018. Prevalence and nature of heating processes in CM and C2-ungrouped chondrites as revealed by insoluble organic matter. Geochimica et Cosmochimica Acta 241, 17-37. doi:10.1016/j.gca.2018.08.029.

Roberts, A., Heslop, D., Zhao, X., Pike, C., 2014. Understanding fine magnetic particle systems through use of first-order reversal curve diagrams. Reviews of Geophysics 52, 557-602. doi:10.1002/2014RG000462.

Roberts, A., Tauxe, L., Heslop, D., Zhao, X., Jiang, Z., 2018. A Critical Appraisal of the "Day" Diagram. Journal of Geophysical Research: Solid Earth 123, 2618-2644. doi:10.1002/2017JB015247.

Rubin, A., Li, Y., 2019. Formation and destruction of magnetite in CO3 chondrites and other chondrite groups. Geochemistry 79. doi:10.1016/j.chemer.2019.07.009.

Rubin, A., Trigo-Rodríguez, J., Huber, H., Wasson, J., 2007. Progressive aqueous alteration of CM carbonaceous chondrites. Geochimica et Cosmochimica Acta 71, 2361-2382. doi:10.1016/j.gca.2007.02.008.

Schrader, D., Davidson, J., 2017. CM and CO chondrites: A common parent body or asteroidal neighbors? Insights from 
chondrule silicates. Geochimica et Cosmochimica Acta 214, 157-171. doi:10.1016/j.gca.2017.07.031.

Schrader, D., Nagashima, K., Krot, A., Ogliore, R., Yin, Q.Z., Amelin, Y., Stirling, C., Kaltenbach, A., 2017. Distribution of $26 \mathrm{Al}$ in the $\mathrm{CR}$ chondrite chondrule-forming region of the protoplanetary disk. Geochimica et Cosmochimica Acta 201, $669275-302$. doi:10.1016/j.gca.2016.06.023.

Schrader, D.L., Davidson, J., McCoy, T.J., Zega, T.J., Russell, S.S., Domanik, K.J., King, A.J., 2021. The Fe/S ratio of pyrrhotite group sulfides in chondrites: An indicator of oxidation and implications for return samples from asteroids Ryugu and Bennu. Geochimica et Cosmochimica Acta 303, 66-91. doi:10.1016/j.gca.2021.03.019.

Singerling, S., Brearley, A., 2018. Primary iron sulfides in CM and CR carbonaceous chondrites: Insights into nebular processes. Meteoritics \& Planetary Science 53, 2078-2106. doi:10.1111/maps.13108.

Singerling, S., Brearley, A., 2020. Altered primary iron sulfides in CM2 and CR2 carbonaceous chondrites: Insights into parent body processes. Meteoritics \& Planetary Science 55, 496-523. doi:10.1111/maps.13450. Suttle, M., King, A., Schofield, P., Bates, H., Russell, S., 2021. The aqueous alteration of CM chondrites, a review. Geochimica et Cosmochimica Acta doi:10.1016/j.gca.2021.01.014.

Thorpe, A.N., Senftle, F.E., Grant, J.R., 2002. Magnetic study of magnetite in the Tagish Lake meteorite. Meteoritics \& Planetary Science 37, 763-771. doi:10.1111/j.1945-5100.2002.tb00853.x.

681 White, L., Tait, K., Langelier, B., Lymer, E., Cernok, A., Kizovski, T., Ma, C., Tschauner, O., Nicklin, R., 2020. EvWhite, L., Tait, K., Langelier, B., Lymer, E., Cernok, A., Kizovski, T., Ma, C., Tschauner, O., Nicklin, R., 2020. Ev-
idence for sodium-rich alkaline water in the Tagish Lake parent body and implications for amino acid synthesis and

683 racemization. Proceedings of the National Academy of Sciences of the United States of America 117, $11217-11219$. 684 doi:10.1073/pnas.2003276117.

685 Yabuta, H., Alexander, C., Fogel, M., Kilcoyne, A., Cody, G., 2010. A molecular and isotopic study of the macromolecular organic matter of the ungrouped C2 WIS 91600 and its relationship to Tagish Lake and PCA 91008. Meteoritics \& Planetary Science 45, 1446-1460 